\title{
Low-Temperature Molten Salt Synthesis for Ligand-Free Transition Metal Oxides Nanoparticles
}

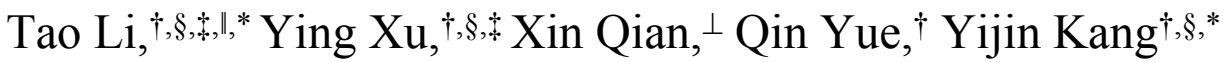 \\ $\dagger$ Institute of Fundamental and Frontier Sciences, University of Electronic Science and
}

Technology of China, Chengdu 610054, R. P. China

$\S$ Institute for Sustainability and Energy, Northwestern University, Evanston, IL 60208, U.S.A

\| School of Physical Science and Technology, Lanzhou University, Lanzhou 730000, P. R. China

${ }^{\perp}$ Department of Materials Science and Engineering, Northwestern University, Evanston, IL, 60208, USA

\$These authors contributed equally

*kangyijin@uestc.edu.cn; lit@1zu.edu.cn 
metal ion

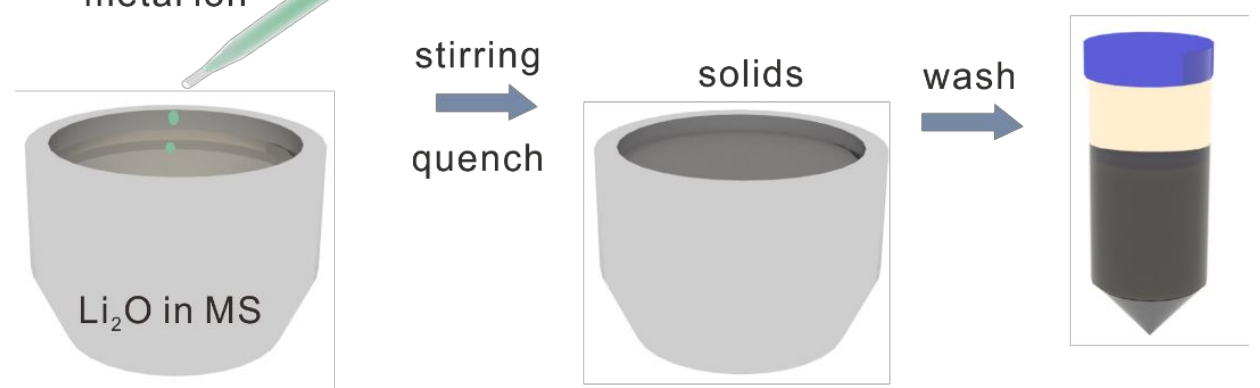

Figure S1. Schematic illustration of TMO synthesis in molten salts.
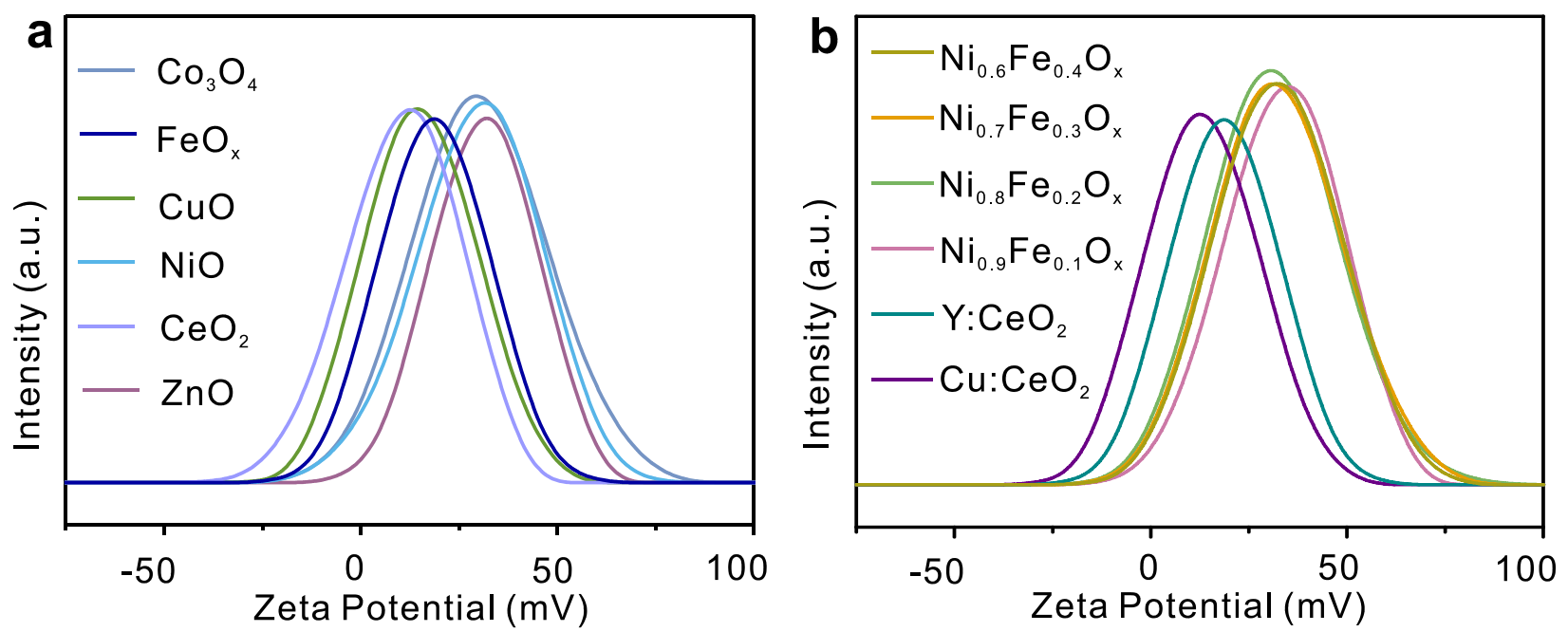

Figure S2. Zeta potentials of as-prepared (a) single metal oxides and (b) doped oxides in ethanol. The positive zeta potential reflects that the metal ions on surface is uncoordinated, ${ }^{1}$ demonstrating no capping agents is attached on the surface of as-prepared TMO NPs.

Table S1. Dopant concentrations of as-prepared nanoparticles by ICP-OES

\begin{tabular}{lcccccc}
\hline \hline & $\mathrm{Cu}: \mathrm{Ce}$ & $\mathrm{Y}: \mathrm{Ce}$ & $\mathrm{Ni}: \mathrm{Fe}(1)$ & $\mathrm{Ni}: \mathrm{Fe}(2)$ & $\mathrm{Ni}: \mathrm{Fe}(3)$ & $\mathrm{Ni}: \mathrm{Fe}(4)$ \\
\hline in product (at\%) & $9.1: 90.9$ & $10.0: 90.0$ & $89.7: 10.3$ & $80.2: 19.8$ & $69.2: 30.8$ & $59.1: 40.9$ \\
in reagent (at\%) & $10: 90$ & $10: 90$ & $90: 10$ & $80: 20$ & $70: 30$ & $60: 40$ \\
\hline
\end{tabular}



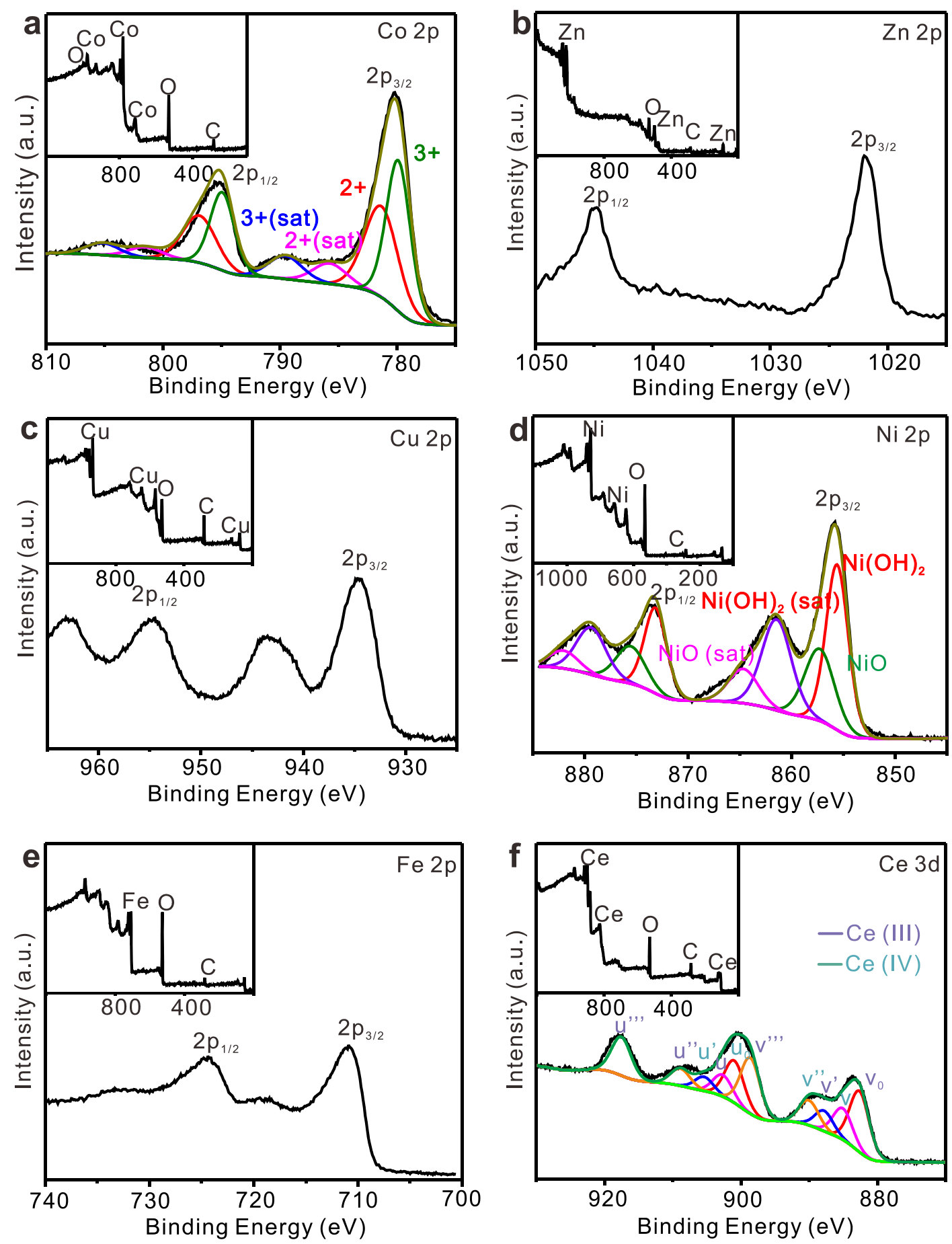

Figure S3. XPS spectra of as-prepared single metal oxides. (a) $\mathrm{Co}_{3} \mathrm{O}_{4}$. (b) $\mathrm{ZnO}$. (c) CuO. (d) $\mathrm{NiO}$. (e) $\mathrm{FeO}_{\mathrm{x}}$. (f) $\mathrm{CeO}_{2}$. XPS was measured to reveal the surface chemical composition of the as-prepared NPs. Particularly, the as-prepared NiO shows a clearly hydroxylated surface state, ${ }^{2}$ which is due to the water wash process. For $\mathrm{CeO}_{2}$, the $\mathrm{Ce}$ (III) and $\mathrm{Ce}$ (IV) coexists, which is a common phenomenon in ceria with oxygen vacancies. ${ }^{3}$ 

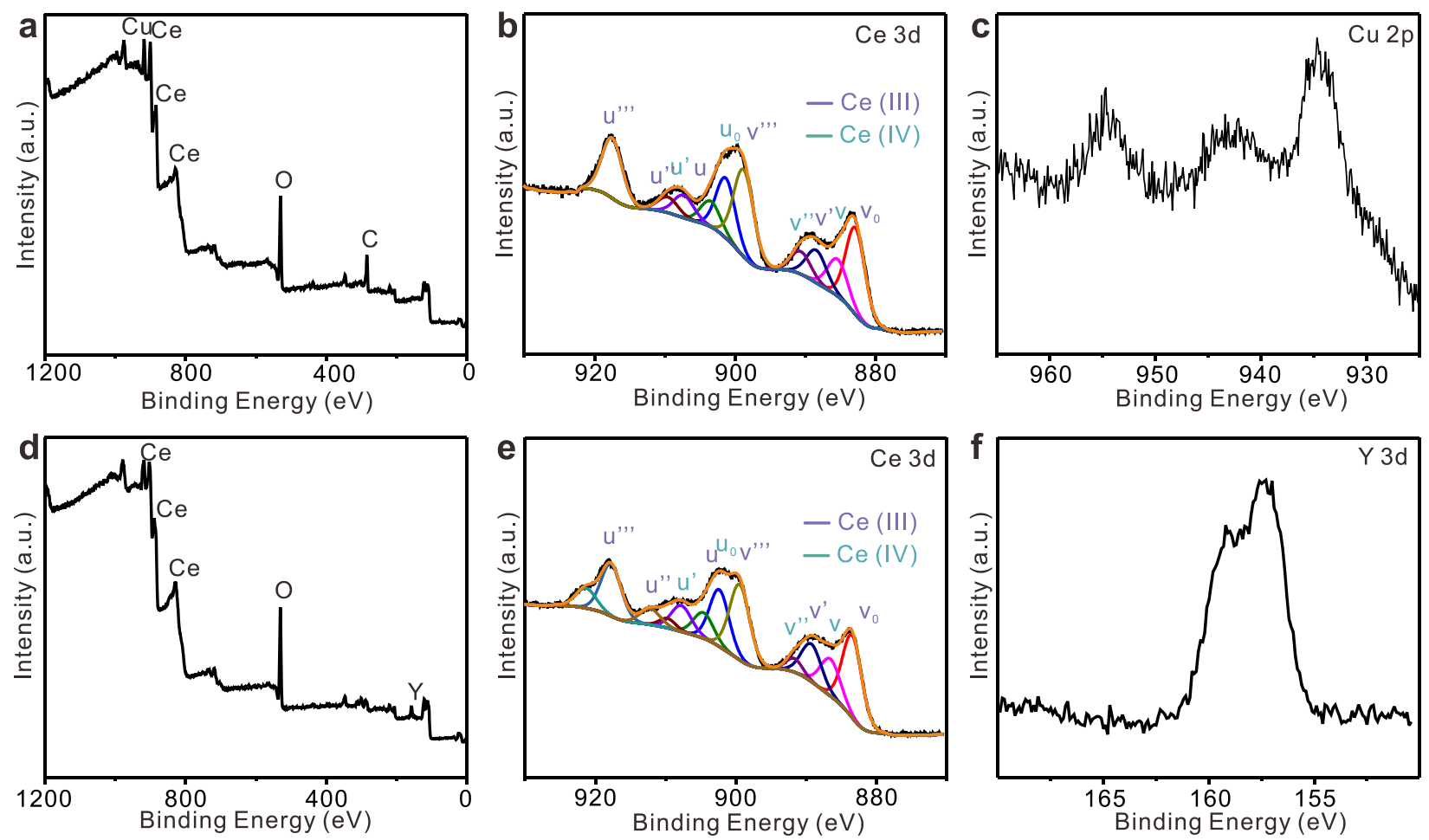

Figure S4. XPS spectra of doped ceria NPs. (a-c) $\mathrm{Cu}$-doped $\mathrm{CeO}_{2}$. (d-f) $\mathrm{Y}$-doped $\mathrm{CeO}_{2}$, suggesting the successful incorporation of doping elements, and there is no alkali metal signal according to the survey spectra in $\mathbf{S 4 a}$ and $\mathbf{S 4 c}$.
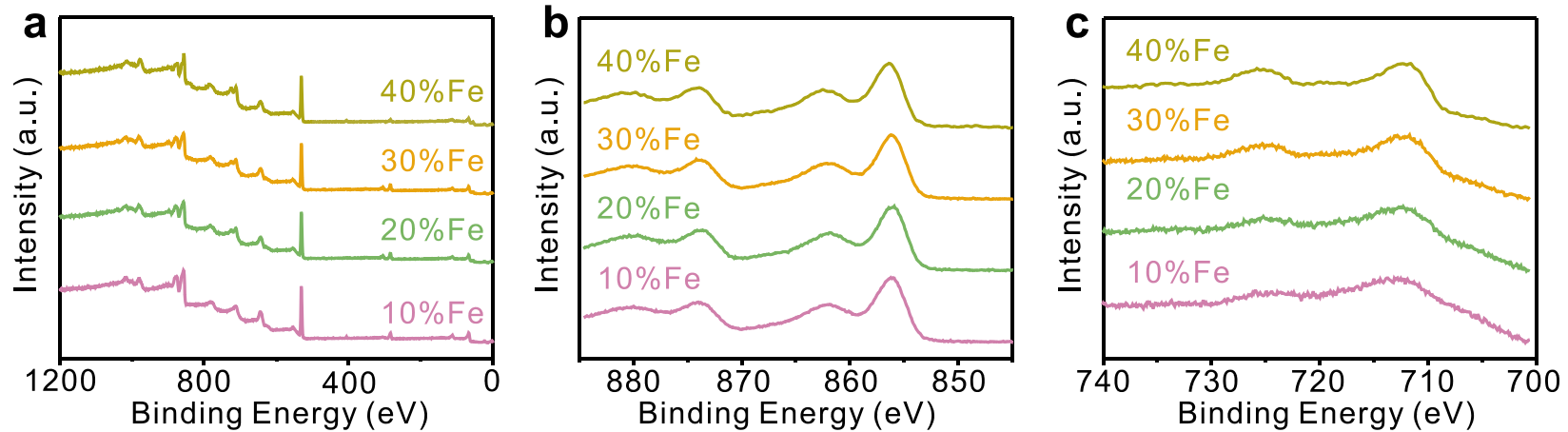

Figure S5. XPS spectra of Ni-Fe-O with different Fe contents. (a) Survey spectra. (b) Ni 2p peaks. (c) Fe 2 p peaks. 


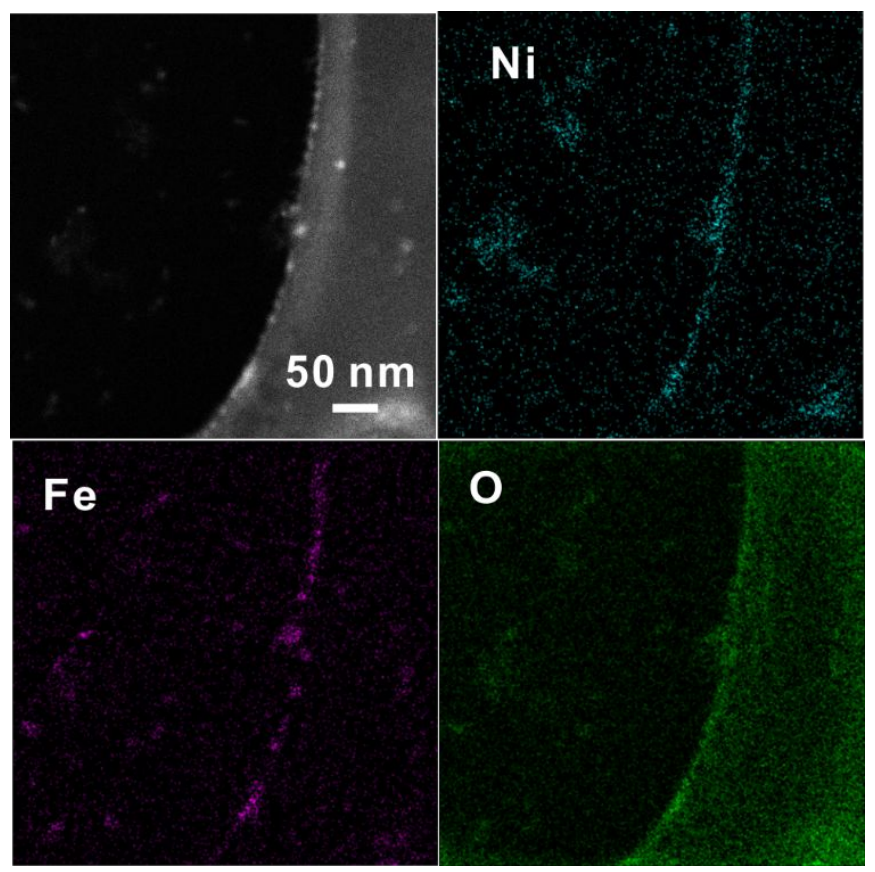

Figure S6. Elemental mapping of $\mathrm{Ni}_{0.7} \mathrm{Fe}_{0.3} \mathrm{O}_{\mathrm{x}}$.
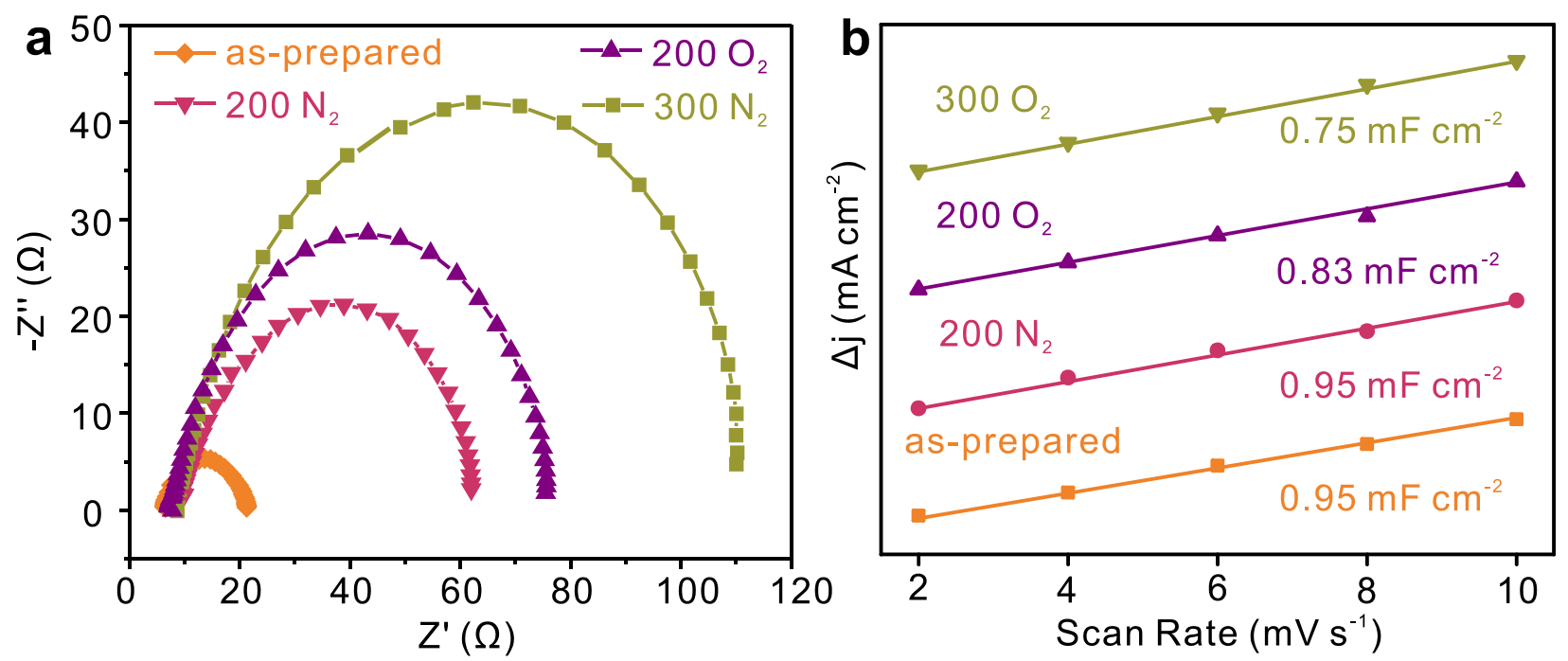

Figure S7. (a) EIS spectra and (b) $\mathrm{C}_{\mathrm{dl}}$ estimated ECSA of samples annealed at different temperature and atmosphere. 


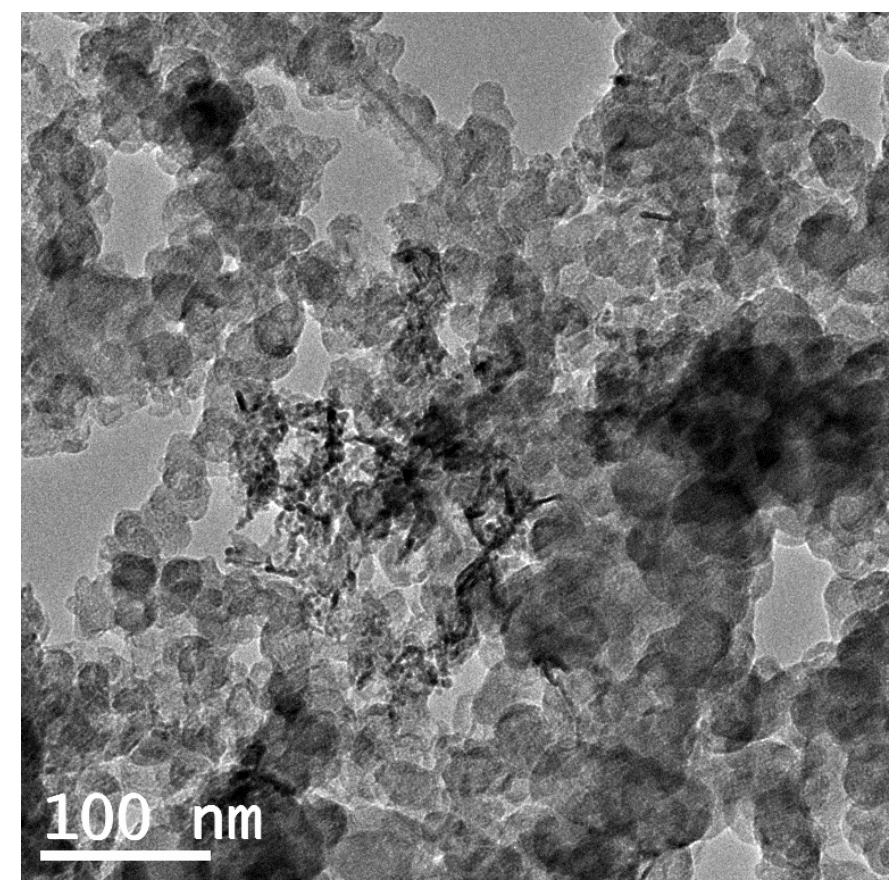

Figure S8. TEM image of $\mathrm{Ni}_{0.7} \mathrm{Fe}_{0.3} \mathrm{O}_{\mathrm{x}} / \mathrm{XC}-72$ annealed at $500{ }^{\circ} \mathrm{C}$.
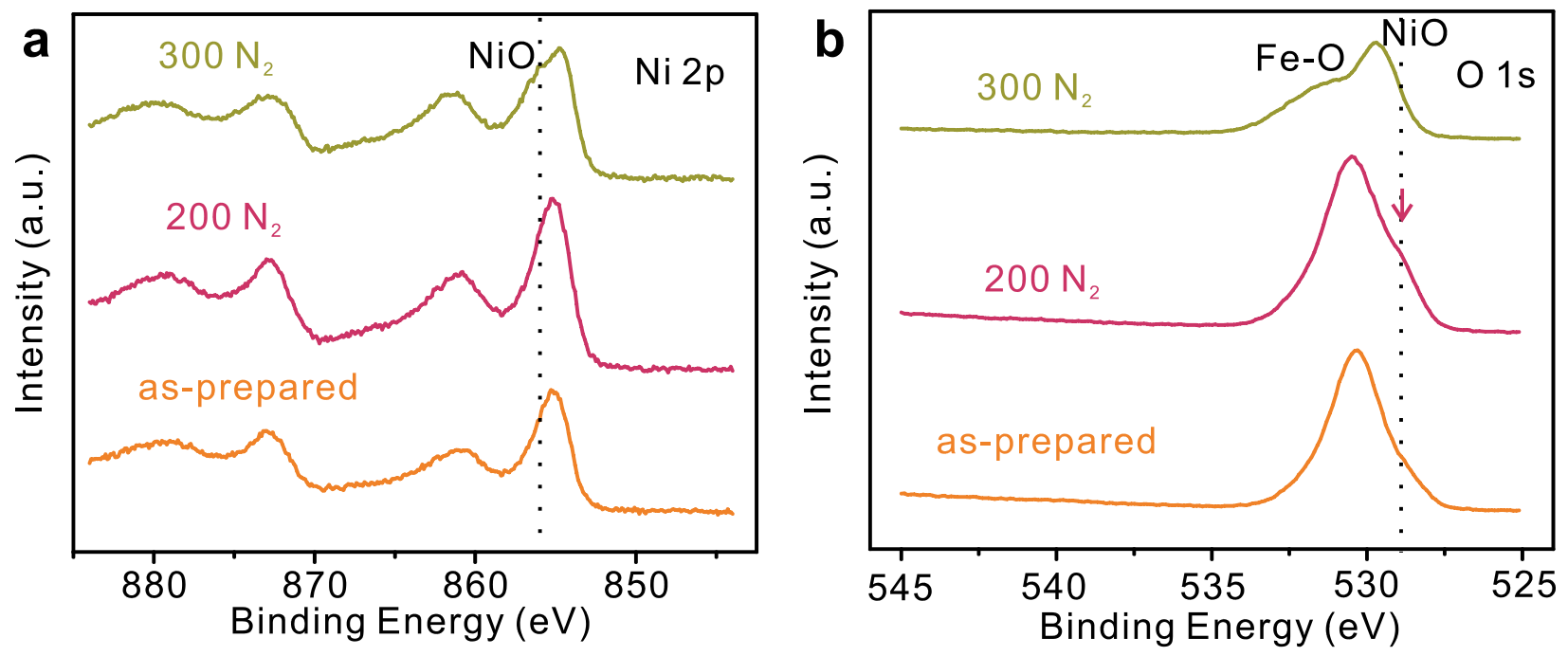

Figure S9. XPS spectra of (a) $\mathrm{Ni} 2 \mathrm{p}$ and (b) $\mathrm{O} 1 \mathrm{~s}$ of $\mathrm{Ni}_{0.7} \mathrm{Fe}_{0.3} \mathrm{O}_{\mathrm{x}}$ annealed at different temperatures. There is a peak around $856.3 \mathrm{eV}$ which can be ascribed to $\mathrm{NiO}$ for sample annealed at $300{ }^{\circ} \mathrm{C}$. The main peak of $531 \mathrm{eV}$ in $\mathbf{S 9 b}$ of sample annealed at $200{ }^{\circ} \mathrm{C}$ could be ascribed to $\mathrm{Ni}(\mathrm{OH})_{2},{ }^{2}$ while the small peak of $529.6 \mathrm{eV}$ is due to the formation of $\mathrm{NiO} .{ }^{4}$ 


\section{REFERENCE}

1. Dong, A.; Ye, X.; Chen, J.; Kang, Y.; Gordon, T.; Kikkawa, J. M.; Murray, C. B. A Generalized Ligand-Exchange Strategy Enabling Sequential Surface Functionalization of Colloidal Nanocrystals. J. Am. Chem. Soc. 2011, 133, (4), 998-1006.

2. Venezia, A.; Bertoncello, R.; Deganello, G. X-Ray Photoelectron Spectroscopy Investigation of Pumice-Supported Nickel Catalysts. Surf. Interface Anal. 1995, 23, (4), 239-247.

3. Curran, C. D.; Lu, L.; Kiely, C. J.; McIntosh, S. Ambient Temperature Aqueous Synthesis of Ultrasmall Copper Doped Ceria Nanocrystals for the Water Gas Shift and Carbon Monoxide Oxidation Reactions. J. Mater. Chem. A 2018, 6, (1), 244-255.

4. Bianchi, C. L.; Cattania, M. G.; Villa, P. XPS Characterization of Ni and Mo Oxides Before and After "in situ" Treatments. Appl. Surf. Sci. 1993, 70, 211-216. 